\title{
Inverted-relief cavity used in VCSELs to suppress higher-order transverse modes
}

\author{
Robert P. Sarzała and Włodzimierz Nakwaski \\ Photonics Group, Institute of Physics, Lodz University of Technology, ul. Wolczanska 219, 90-924 Lodz, Poland
}

Received August 21, 2013; accepted February 11, 2014; published March 31, 2014

\begin{abstract}
One of the most acute VCSEL disadvantages is the excitation of higher-order transverse modes in devices emitting more intense radiation. The paper presents the results of simulation of VCSEL performance with the aid of an inverted-relief cavity used to suppress those modes. This suppression method is very efficient and relatively simple from a technological point of view, so it is worth recommending.
\end{abstract}

Vertical-cavity surface-emitting diode lasers (VCSELs) have recently forced out hitherto generally used edge-emitting diode lasers from most of their earlier applications because of VCSEL stable inherent singlelongitudinal-mode operation, low price and low-divergent, circularly symmetric output beam without astigmatism. Besides, VCSEL manufacturing is relatively inexpensive because imperfect structures may be revealed and removed before epitaxial wafers are cut into individual devices. However, the single-fundamental-mode (SFM) operation expected in most of the VCSEL applications is limited to devices emitting only relatively low output powers [1]. Such a behavior is a consequence of excitation of higher-order transverse $\mathrm{LP}_{\mathrm{ij}}$ modes with an increase in VCSEL output. Therefore wider VCSEL applications could be possible if effective methods [2] used to suppress these modes were discovered and applied. In the present paper, the efficiency of a promising simple surface-relief suppression method is investigated theoretically and the threshold performance of VCSELs using this method is discussed. The simulation has been carried out for the room-temperature (RT) continuouswave $(\mathrm{CW})$ performance of the above laser with the aid of a three-dimensional optical-electrical-thermal recombination self-consistent VCSEL threshold model [3].

The standard GaAs-based OC VCSEL structure under consideration designed for the $1.3-\mu \mathrm{m}$ emission is shown in Fig. 1. The output beam is emitted through a hole etched in an upper $p$-side metal contact. The $n$-side contact covers the whole laser base. One-wavelength optical cavity is formed between distributed-Braggreflector (DBR) GaAs/AlGaAs mirrors. Radial current confinement has been created with the aid of a radial oxidation from outside towards the laser axis of the upper

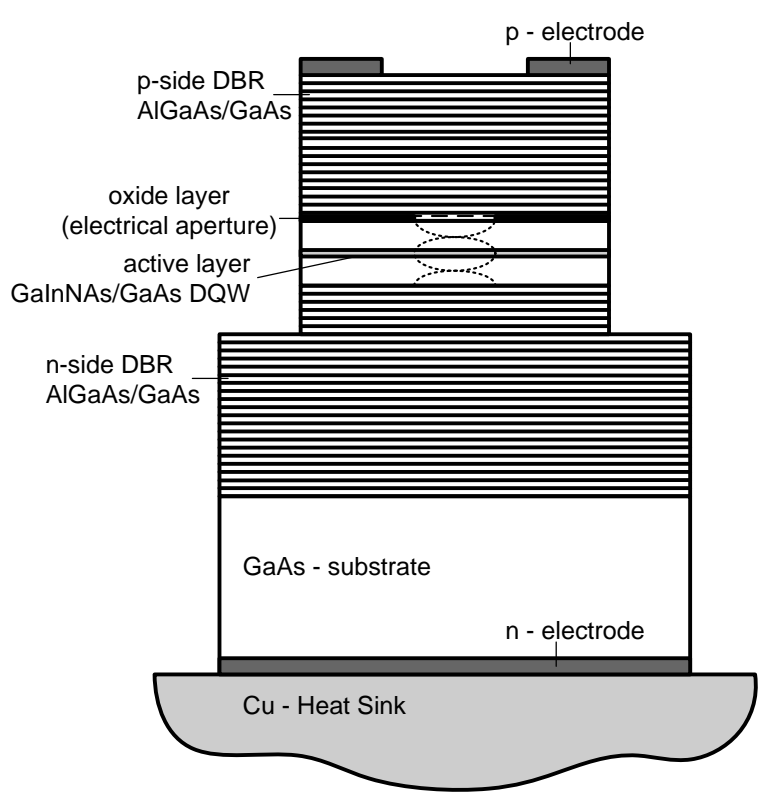

Fig. 1. Nominal structure of a VCSEL under consideration.

DBR AlGaAs layer of a relatively high AlAs mole fraction. In this way, an annular $\mathrm{Al}_{\mathrm{x}} \mathrm{O}_{\mathrm{y}}$ oxide layer of relatively high electrical resistivity is created and the current path is effectively confined to the unaffected circle of a radius equal to an internal radius of the annular oxide. Besides the applied GaInNAs/GaAs quantum-well (QW) active region requires some detuning to reach the desired $1.3-\mu \mathrm{m}$ emission. Then higher-order transverse modes exhibit distinctly higher optical gain than the lowest ones, including the fundamental mode. It makes the problem of reaching the SFM operation in this device even more difficult.

For the standard VCSEL design with an active-region radius $r_{\mathrm{A}}$ of $6 \mu \mathrm{m}$, its RT $\mathrm{CW}$ threshold parameters, i.e. lasing wavelengths $\lambda$, maximal optical gain $g_{\max }$ within the active region and imaginary wavelength $\lambda_{\text {im }}$, determined for a supplying voltage of $1.52 \mathrm{~V}$, are listed in Table $1 . \lambda_{\text {im }}$ has negative values below the lasing threshold, becomes equal to zero for a lasing threshold and positive above. As one can see, in this case, the lasing threshold is reached only by the $\mathrm{LP}_{51}$ and $\mathrm{LP}_{61}$ higher-order transverse modes. 
Mirror reflectivity in lateral VCSEL sections may be seemingly reduced with the aid of a ring-shaped pattering around the central mirror part. Then, after a careful etching of this area, i.e. when the etch depth is equal to $\lambda /\left(4 n_{\mathrm{R}, \mathrm{eff}}\right)$, where $n_{\mathrm{R}, \text { fff }}$ is the mode effective index of refraction, the resultant surface relief VCSEL structure leads in this region to the out-of-phase optical wave reflection at the semiconductor-air interface. Hence, the optical field within the VCSEL cavity behaves as if it met a reduced mirror reflectivity beyond the central part of the VCSEL mirror. It creates a relatively high mirror loss contrast between the mirror etched area and the un-etched central area, which leads to increasing mirror losses of higher-order transverse modes [4-6] penetrating this etched mirror part more effectively than the fundamental one.

Table 1 . Wavelength $\lambda$, maximal optical gain $g_{\max }$ and the imaginary part of the wavelength $\lambda_{\mathrm{im}}$ of successive transverse $\mathrm{LP}_{\mathrm{ij}}$ modes determined for the standard OC VCSEL (Fig. 1) under consideration with $12-\mu \mathrm{m}$ diameter active region supplied with the voltage of $1.52 \mathrm{~V}$.

\begin{tabular}{|c|c|c|c|}
\hline \multirow{2}{*}{ Mod } & \multirow{2}{*}{$\begin{array}{c}\lambda \\
{[\mathrm{nm}]}\end{array}$} & $\begin{array}{c}g_{\max } \\
{\left[\mathrm{cm}^{-1}\right]}\end{array}$ & $\lambda_{\text {im }}$ \\
\hline $\mathrm{LP}_{01}$ & 1300.15 & 1382 & $-8.68 \mathrm{e}-10$ \\
\hline $\mathrm{LP}_{11}$ & 1299.63 & 1442 & $-6.61 \mathrm{e}-10$ \\
\hline $\mathrm{LP}_{21}$ & 1298.95 & 1519 & $-4.30 \mathrm{e}-10$ \\
\hline $\mathrm{LP}_{31}$ & 1298.13 & 1609 & $-1.99 \mathrm{e}-10$ \\
\hline $\mathrm{LP}_{41}$ & 1297.18 & 1704 & $-0.16 \mathrm{e}-10$ \\
\hline $\mathbf{L P}_{\mathbf{5 1}}$ & $\mathbf{1 2 9 6 . 0 9}$ & $\mathbf{1 8 0 5}$ & $\mathbf{1 . 0 4 e}-10$ \\
\hline $\mathrm{LP}_{61}$ & 1294.88 & 1902 & $0.78 \mathrm{e}-10$ \\
\hline $\mathrm{LP}_{71}$ & 1293.55 & 1989 & $-1.64 \mathrm{e}-10$ \\
\hline
\end{tabular}

a)

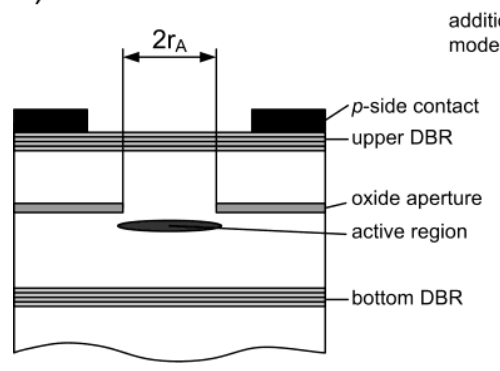

b) additional layer responsible for

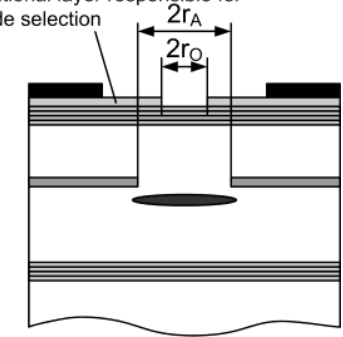

Fig. 2. The standard VCSEL structure (a) and the structure with the inverted relief in the top mirror (b), where: $r_{A}$ - radius of the hole within the oxide layer, $r_{\mathrm{o}}$ - radius of the mode-selection structure.

Even more interesting is an analogous method known as the inverted-relief technique (Fig. 2). Its main idea consists in growing an extra $\lambda /\left(4 n_{\mathrm{R}, \mathrm{eff}}\right)$-thick layer on the top of the output DBR structure and complete etching a circular disk-shaped structure through this layer in the DBR centre. In this structure, optical waves reflected at the topmost layer/air interface within the central etched disk and those reflected outside will be in anti-phase. Therefore only the reflection of the fundamental mode, mostly confined within the central etched area, will be nearly completely restored, whereas higher-order modes will experience both reduced reflectivity and additional absorption in the extra $\lambda /\left(4 n_{\mathrm{R}, \text { eff }}\right)$-thick layer outside the central area.

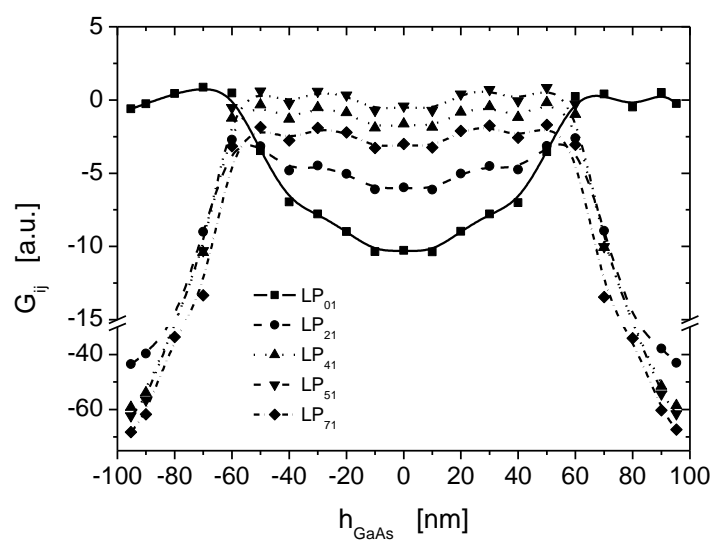

Fig. 3. Modal gain $G_{\mathrm{ij}}$ of successive $i j$-th transverse modes as a function of a thickness $h_{\mathrm{GaAs}}$ of the etched (negative values) or the extra (positive values) parts of the additional GaAs DBR layer for $r_{\mathrm{A}}=12 \mu \mathrm{m}$ and $r_{\mathrm{o}}=7 \mu \mathrm{m}$ (see Fig. 2).

The impact of the reduced thickness of an additional GaAs layer within the central DBR part on modal gain values of successive transverse modes is shown in Fig. 3. Surprisingly, the curves are nearly symmetrical with respect to the $h_{\mathrm{GaAs}}=0$ value, which means that both etching and adding an additional layer in a DBR structure have a similar impact on modal gain. A steady increase in the etched thickness results in an increase in the modal $G_{01}$ gain of the fundamental $\mathrm{LP}_{01}$ mode from its negative value determined for too small $h_{\mathrm{GaAs}}$. As expected, $G_{01}$ becomes positive for $h_{\mathrm{GaAs}}$ values close to $\lambda /\left(4 n_{\mathrm{R}, \mathrm{eff}}\right) \approx 95.3 \mathrm{~nm}$.

A very stable SFM operation has been found for the VCSEL structure shown in Fig. $2 b$ with the same $12-\mu \mathrm{m}$ diameter active region as the nominal VCSEL (Fig. 2a). It is clearly seen in Table 2 (analogous to Table 1), where there are listed the wavelength $\lambda$ of emitted radiation, maximal optical gain $g_{\max }$ and imaginary wavelength part $\lambda_{\text {im }}$ of successive transverse LP $_{\mathrm{ij}}$ modes determined at RT for such a VCSEL with a central opening of diameter equal to $7 \mu \mathrm{m}$ and supplied with $1.78 \mathrm{~V}$. As one can see, the VCSEL under consideration exhibits an excellent selection of higher-order transverse modes, which is proved by very negative values of imaginary wavelength parts $\lambda_{\text {im }}$ of all transverse $\mathrm{LP}_{\mathrm{ij}}$ modes except those of the fundamental $\mathrm{LP}_{01}$ one.

The structure shown in Fig. 2b demonstrates quite essential changes as compared with the nominal structure. 
Its maximal values of the active-region threshold current, threshold voltage and temperature have been dramatically increased. Therefore it is evident that the excellent selection of higher-order transverse modes obtained in this laser has been achieved at the cost of an increase in radiation losses. This increase influences not only these modes, as expected, but also, although to a much lower extent, the fundamental mode.

Table 2. Wavelength $\lambda$, maximal optical gain $g_{\max }$ and the imaginary part of the wavelength $\lambda_{\mathrm{im}}$ of successive transverse $\mathrm{LP}_{\mathrm{ij}}$ modes determined for the inverted-relief VCSEL structure shown in Fig. 2b. The considered OC VCSEL is supplied with a voltage of $1.78 \mathrm{~V}$ and is equipped with the $12-\mu \mathrm{m}$ diameter active region. Its DBR mirror contains an additional GaAs layer of a thickness equal to $\lambda /\left(4 n_{\mathrm{R}, \text { eff }}\right)$, where $n_{\mathrm{R}, \text { eff }}$ is the mode effective index of refraction, and its radiation is going out through a central etched opening of $7-\mu \mathrm{m}$ diameter.

\begin{tabular}{|c|c|c|c|}
\hline \multirow{2}{*}{ Mod } & \multirow{2}{*}{$\begin{array}{c}\lambda \\
{[\mathrm{nm}]}\end{array}$} & $\begin{array}{c}|c| \\
g_{\text {max }} \\
{\left[\mathrm{cm}^{-1}\right]}\end{array}$ & $\lambda_{\text {im }}$ \\
\hline $\mathbf{L P}_{\mathbf{0 1}}$ & $\mathbf{1 3 0 0 . 4 2}$ & $\mathbf{2 0 5 8}$ & $\mathbf{1 . 3 4 e - 1 0}$ \\
\hline $\mathrm{LP}_{11}$ & 1299.89 & 2126 & $-24.19 \mathrm{e}-10$ \\
\hline $\mathrm{LP}_{21}$ & 1299.21 & 2210 & $-41.36 \mathrm{e}-10$ \\
\hline $\mathrm{LP}_{31}$ & 1298.39 & 2304 & $-51.44 \mathrm{e}-10$ \\
\hline $\mathrm{LP}_{41}$ & 1297.43 & 2403 & $-56.82 \mathrm{e}-10$ \\
\hline $\mathrm{LP}_{51}$ & 1296.34 & 2501 & $-59.79 \mathrm{e}-10$ \\
\hline $\mathrm{LP}_{61}$ & 1295.12 & 2592 & $-62.16 \mathrm{e}-10$ \\
\hline $\mathrm{LP}_{71}$ & 1293.79 & 2672 & $-65.46 \mathrm{e}-10$ \\
\hline
\end{tabular}

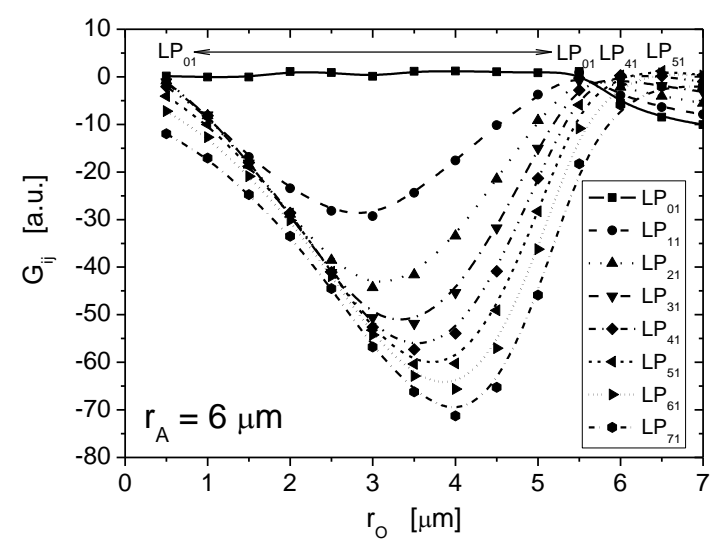

Fig. 4. Values of a modal gain $G_{\mathrm{ij}}$ of successive $\mathrm{LP}_{\mathrm{ij}}$ transverse modes excited at RT in the nominal VCSEL and equipped with an additional mechanism of mode selection shown in Fig. $2 \mathrm{~b}$ as a function of the radius $r_{0}$ of the opening etched in the additional DBR layer of thickness $\lambda /\left(4 n_{\mathrm{R}, \mathrm{eff}}\right)$.

Let us now find an optimal diameter of the opening etched through the additional $\lambda /\left(4 n_{\mathrm{R}, \mathrm{eff}}\right)$-thick layer of the output mirror of the VCSEL design with an inverted relief. The modal gains $G_{\mathrm{ij}}$ of successive transverse modes $\mathrm{LP}_{\mathrm{ij}}$ are plotted in Fig. 4 as a function of the radius $r_{0}$ of this opening. As one can see, a suitable $r_{0}$ choice enables an excellent selection of transverse modes. For all $r_{0}$ values between $2 \mu \mathrm{m}$ and $4.5 \mu \mathrm{m}$, especially for $r_{0}=3 \mu \mathrm{m}$, the modal gain $G_{01}$ of the fundamental $\mathrm{LP}_{01}$ mode is decidedly higher than modal gains of all higher-order transverse modes. In oxide-confined devices, an increase in the opening diameter results in a more non-uniform gain distribution within the VCSEL active region, distinctly higher close to the active-region edge than that within its broad central part. It gradually increases the modal gains of transverse modes of intensity distributions shaped similarly. Finally, for $r_{0}=r_{\mathrm{A}}$, higher-order transverse modes exhibit higher modal gains than those of the fundamental mode, which results in a distinctly multimode VCSEL operation. The above conclusions remain valid also for $r_{0}$ values between $3.5 \mu \mathrm{m}$ and $7 \mu \mathrm{m}$, especially for $r_{0}=4.5 \mu \mathrm{m}$, for which the modal gain $G_{01}$ of the fundamental $\mathrm{LP}_{01}$ mode is decidedly higher than modal gains of all higher-order transverse modes.

The authors would like to acknowledge the partial support from the Polish Ministry of Science and Higher Education (MNiSzW), grant No NN 515417635 and project: "A Novel Photonic Crystal Surface Emitting Laser Incorporating a High-Index-Contrast Grating" from Polish National Centre for Research and Development.

\section{References}

[1] C. Jung, R. Jäger, M. Grabherr, P. Schnitzer, R. Michalzik, B. Weigl, S. Müller, K.J. Ebeling, Electron. Lett. 33, 1790 (1997).

[2] W. Nakwaski, Opto-Electr. Rev. 19, 119 (2011).

[3] R. Sarzała, T. Czyszanowski, M. Wasiak, M. Dems, Ł. Piskorski, W. Nakwaski, K. Panajotov, Adv. in Opt. Techn. (2012)- article ID 689519.

[4] H. Martinsson, J.A. Vukušič, A. Larsson, IEEE Photon. Technol. Lett. 12, 1129 (2000).

[5] H.J. Unold, S.W.Z. Mahmoud, R. Jäger, M. Grabherr, R. Michalzik, K.J. Ebeling, IEEE J. Sel. Top. Quantum Electron. 7, 386 (2001).

[6] D. Supper, J. Pfeiffer, M. Ilzhöfer, Ch. Giuliani, Ch. Degen, A.P. Lima, A. Ramakrishnan, G. Ebbinghaus, G. Steinle, Opt. Comm. 267, 447 (2006) 